\title{
Effect of Electroacupuncture at Auricular Acupoints "Heart" and "Brainstem" in Improving the Consciousness of Patients after Traumatic Brain Injury: Study Protocol for a Randomized Controlled Trial
}

Tong Liu ( $\square$ liutongmama@163.com )

Guangdong Second Hospital of TCM

Jia-Ni Yu

Guangdong Second Hospital of TCM

Wei-chuan Kuang

Guangdong Second Hospital of TCM

Xiao-yin Wang

Guangdong Second Hospital of TCM

Ye Jiang

Guangdong Second Hospital of TCM

Xi Wen

Guangdong Second Hospital of TCM

Xiao-jia Qiu

Guangdong Second Hospital of TCM

\section{Method Article}

Keywords: Auricular acupoint, Electro-acupuncture, Body acupuncture, Traumatic brain injury, Consciousness, GCS, Mismatch negativity

Posted Date: January 14th, 2019

DOI: https://doi.org/10.21203/rs.2.209/v1

License: (c) (1) This work is licensed under a Creative Commons Attribution 4.0 International License.

Read Full License 


\section{Abstract}

Background:Traumatic brain injury (TBI) has become a major cause of morbidity and mortality both in China and abroad. Disorders of consciousness (DOC) following severe traumatic brain injury (TBI) is a common refractory complication, resulting in difficult rehabilitation and poor life quality. However, effective therapeutic approaches remain limited. Although auricular-acupuncture has been widely applied in the treatment of neurological disorders in China, its efficacy and safety for consciousness recovery remain to be elucidated.

Methods: Here, we conduct a study design and protocol of a randomized, blinded, controlled study to evaluate the efficacy and safety of Electroacupuncture at Auricular Acupoints "Heart" and "Brainstem" in the consciousness recovery of patients with TBI. A total of 80 patients with initial Glasgow coma scale score between 3-8 points will be recruited in the trial and randomized into treatment (combined application of auricular and body acupuncture) group or control (body acupuncture alone) group. Patients in the treatment group will receive electroacupuncture at bilateral auricular acupoints "Heart" and "Brainstem" (four points in total) combined with body acupuncture in a supine position while patients in the control group will receive body acupuncture alone for 4 weeks. The primary outcomes are changes of Glasgow coma scale score and mismatch negativity of event-related brain potentials before and after treatment. The secondary outcome measures will be changes of Barthel and FuglMeyer scores. The safety will also be assessed by monitoring the incidence of adverse events and changes in vital signs during the study.

Discussion: Results from this trial will significantly support the application of auricular acupuncture in the consciousness recovery of patients with severe TBI. If found to be effective and safe, auricular acupuncture will be a valuable complementary option for comatose patients with TBI.

Trial registration: Chinese Clinical Trial Registry: ChiCTR1800020245. Registered on 21 December 2018.

Keywords: Auricular acupoint, Electro-acupuncture, Body acupuncture, Traumatic brain injury, Consciousness, GCS, Mismatch negativity

\section{Background}

Traumatic brain injury (TBI) is a leading cause of morbidity and mortality among children and young adults[1]. In China, it is reported that the mortality rate induced by TBI was among $2.7 \%$ to $21.8 \%$ [2]. Although the number of patients survived from severe TBI increased gradually accompanied by the advance in neurocritical care, many survivors still failed to fully recover a state of disorders of consciousness (DOC) such as coma, vegetative state, and minimally conscious state[3]. The neuropathology of DOC has been extensively described at postmortem and diffuse disruption of subcortical white matter was reported the most common reasons found in victims of TBI induced DOC[46]. Longer time to recovery from DOC is predictive of worse prognosis after TBI[7-8], and shortening the period of DOC may be helpful in improving outcomes, at least partly increase participation in 
rehabilitation treatment[9-10]. However, only few limited strategies have been found for the treatment of DOC.

As a complementary and alternative therapy, acupuncture is an important component of traditional Chinese medicine and has been widely used in various diseases during the past thousands of years in China. Many types are involved in acupuncture treatment, such as body acupuncture, auricular acupuncture, scalp acupuncture, wrist-ankle acupuncture, etc. Body acupuncture has been proved in improving consciousness induced by TBI in many studies[11-12]. Auricular acupuncture has also been accepted in many disorders or conditions, such as pain [13], constipation[14], addiction[15], insomnia[16], cognitive impairment and dementia[17], however, its effect for DOC has not been examined.

Thus, we present a study design and protocol of a randomized, blinded, controlled study to assess the efficacy and safety of auricular electroacupuncture combined with body acupuncture in improving the consciousness in patients with TBI. The objetive of this study was to determine if auricular acupuncture combined with body acupuncture is more effective than body acupuncture alone. The results of this study are expected to establish an optimal acupuncture procedure for DOC.

\section{Methods}

This study is a single-center, randomized controlled, assessor-blinded clinical trial which was devised following the Consolidated Standards of Reporting Trials (CONSORT) Statement recommendations[18]. This study has been approved by the Ethical Committee of GuangDong Second Hospital of Traditional Chinese Medicine. We registered the study on the Chinese Clinical Trial Registry with registration number ChiCTR180002024.

It will be performed in the GuangDong Second Hospital of Traditional Chinese Medicine. 80 patients who meet the eligibility criteria and sign an informed consent form will be randomly devided into two groups to receive either auricular point electroacupuncture plus body acupuncture or body acupuncture alone in a 1:1 ratio. All the participants will accept conventional treatment, including the prescription of coma arousal and neuroprotective medicine. Acupuncture will be performed 30 min per day, 5 days per week for 4 weeks. Doctors with more than 5 years of clinical experience will be allowed to perform the interventions. After 4 weeks, Outcome measurements including the Glasgow Coma Scale (GCS) $\square \mathrm{MMN} \square$ Barthel and FuglMeyer scores will be analysed by doctors who was blind to the allocation of the patients. The flowchart of the trial is shown in Figure 1.

\section{Participants}

\section{Recruitment strategies}

Inpatients at acupuncture departments in GuangDong Second Hospital of Traditional Chinese Medicine will be recruited mainly in this RCT. Additionally, posters will be put in the hospitals and a Chinese multipurpose social media named WeChat will be applied to recruit. Brief descriptions of eligible criteria, 
the free acupuncture treatments and the possible risks of the trial will be marked and all patients' family members have the right to participate or drop out at any time, and will be required to sign the informed consent before the trial begin.

Eligibility criteria: inclusion criteria

The inclusion criteria will be as follows.

1.Meet the diagnostic criteria for TBI;

2.Aged 18-70 years old, male or female;

3. Initial GCS score between 3-8 points on admission and lasted more than 2 weeks;

4. Imaging (MRI) head has no obvious shift, missing, large necrosis of brain structure change and obvious brain stem (not including pyramidal tract) or thalamic lesions, each lobe lesions range can not exceed $30 \%$ of the scope of one side of the brain;

5.No primary consciousness disorder and limb functional activity disorder;

6.Signed informed consent.

Eligibility criteria: exclusion criteria

The exclusion criteria will be as follows.

1.DOC not induced by TBl;

2.Clinical course more than 1 year;

3.Combination of heart, liver or kidney failure endanger the safety of life at any time;

4.Younger than 18 years or older than 70 years;

5.Those who do not receive electroacupuncture therapy;

6.Women with pregnancy and lactation.

Randomization and allocation

Random numbers will be generated by the random number generator in the SPSS statistical software package (Version 22.0, SAS Institute Inc.). Another specified researcher who is not involved in the study was responsible for it.

The allocation of participants will be sealed in a sequentially numbered opaque envelope. If the participant meets the inclusion criteria and signs informed consent, the above specified researcher will sequentially give the sealed random number envelope to the physician, who will open the envelope and 
allocate the participant to either auricular point electroacupuncture plus body acupuncture group or body acupuncture alone group according to the random number.

\section{Blinding}

Because of the add-on study design, a single-blinded method will be used. While the participants and practitioners cannot be blinded, we blinded the outcome assessors, data manager, and statistics analyzer. The assessor will be instructed not to communicate with participants about the possibility of their treatment. James et al.'s blinding index will be evaluated after the completion of the study to evaluate the success of blinding[19].

Intervention

Study schedule

Treatment group intervention

Patients in the treatment group will receive both auricular point electroacupuncture and body acupuncture in a supine position. Auricular point electroacupuncture manipulation was as follows: Following disinfection of skin with $75 \%$ alcohol, bilateral auricular acupoints "Heart" and "Brainstem" [20] (four points in total) will be stimulated using disposable and stainless needles $(13 \mathrm{~mm}$ long and $0.25 \mathrm{~mm}$ in diameter, Suzhou Huanqiu Acupuncture Medical Appliance, Suzhou, China) inserted to a depth of $2 \mathrm{~mm}$, and connected to an electrical stimulator (SDZ-V EA; Huatuo, Suzhou, China) with a frequency of $2 / 10 \mathrm{~Hz}$ and current of $1 \mathrm{~mA}$. Body acupuncture manipulation was as follows: After disinfection, GV26[21] was inserted towards the nose at a depth of $10 \mathrm{~mm}$ while bilateral PC6 ${ }^{[21]}$ and SP6 ${ }^{[21]}$ was perpendicularly inserted at a depth of $20 \mathrm{~mm}$ by disposable and stainless needles (40 $\mathrm{mm}$ long and $0.30 \mathrm{~mm}$ in diameter, Suzhou Huanqiu Acupuncture Medical Appliance, Suzhou, China). The needle will be manipulated using twirling, lifting, thrusting, and mild reinforcing-reducing methods to promote Qi. Eye moisture or the presence of tears will be used as an indicator of Qi arrival since patients are not able to express their feelings. Both the two acupuncture method was restrained for $30 \mathrm{~min}$, once daily, 5 times a week and lasted for 4 weeks. The location of acupoints applied was shown in Table1.

Control group intervention. The control group patients will receive body acupuncture treatment following the same protocol used for the treatment group. Auricular point electroacupuncture will not be administered.

\section{Outcome measures $\llbracket$}

The primary efficacy endpoint will be the Glasgow Coma Scale (GCS) and MMN at week 8. Secondary measurements will be changes of Barthel and FuglMeyer scores. Safety will be assessed by monitoring adverse events as well as changes in vital signs during the study. All the patients will be followed up at three months by a clinic visit or telephone survey to evaluate GCSロBarthel and FugIMeyer scores. (see 
Fig. 2 and Additional file 1: Standard Protocol Items: Recommendations for Interventional Trials (SPIRIT) Checklist).

Safety assessment

Any adverse events, including acupoint hematoma, infection, dizziness and apostasies will be evaluated during the whole treatment by the researcher. If any severe adverse events occurring, acupuncture intervention will be ceased immediately and the principal investigator will be informed to take proper actions. 1 month will be followed up after the trial in case of subsequent adverse events.

Sample size calculation

The trial is designed to determine the role of Electroacupuncture at "Heart" and "Brainstem" auricular point combined with conventional acupuncture in the consciousness recovery of patients with TBI and prove that Electroacupuncture at "Heart" and "Brainstem" auricular point combined with conventional acupuncture is superior to conventional acupuncture alone. Therefore, GCS change will be used as an evaluation index. According to our previous pilot study, the change of GCS before and after treatment in the treatment group was shown to be $5.62 \pm 1.41(n=8)$ and that in control group was $4.50 \pm 1.85(n=8)$. Sample size was estimated using the following formula:

where $n$ represents the number of samples required, $n=n_{1}+n_{2}, Q_{1}=n_{1} / n, Q_{2}=n_{2} / n$ with a significance level $(a=0.05)$ of a two-sided two-sample t-test and $80 \%$ power to detect a difference between the two groups. Thus, a total sample size of 80 patients will be recruited allowing for $10 \%$ of attrition, with 40 in each.

Statistical analysis

SPSS 20.0 (IBM SPSS Statistics, IBM Corp, Somers, New York, USA) will be used to analyze the data. Quantitative data will be presented as mean \pm SD. GCS $\square$ Barthel and FuglMeyer scores will be conducted between the two groups by a superiority independent sample t-test with a $95 \% \mathrm{Cl}$. The incidence of complications and adverse events will be compared by $\chi 2$ test. A mixed model procedure will be applied to compare longitudinal changes in MMN amplitude between the two groups. Any missing data will be replaced by the last mea- sured value. For all analyses, $P$ values of less than 0.05 will be considered statistically significant.

Quality control

Before the trial, all the acupuncturists $\square$ nurses and assessors will be trained strictly in order to guarantee homogeneity in the measurement data and ensure high-quality data results. The training content will include study protocol, diagnosis, inclusion and exclusion criteria, recording method of CRF, the location of the acupoints, acupuncture and auricular acupuncture operation techniques, disposal of bleeding. All the study data will be recorded on the case report form. Dropouts and withdrawals from the study will be recorded in detail based on the intervention and follow-up periods. Data will be uploaded and verified by 
other two researcher who were not involved in the trial. This trial will be monitored by the Scientific Research Department of GuangDong Second Hospital of Traditional Chinese Medicine.

\section{Discussion}

The incidence of TBI has become more common with the rapid societal development and increase in traffic accidents[22-23]. It affects at least 1.7 million individuals in the United States and causing 1.5 million hospitalizations in the European Union yearly[24-26]. It is also a serious public health problem in China and causes a substantial number of deaths and temporary and permanent disabilities[27]. Even if survives, many patients will fall into a long period of DOC. Thus, it is urgent to found more effective and safe strategies to improve the outcome of patients with DOC.

Acupuncture has been widely applied in China and abroad to treat neurological diseases, its effect for DOC has also been certified by some studies[28-29]. Here, we conducted a study to compare the effect of auricular combined with body acupuncture to body acupuncture alone in order to clarify if auricular acupuncture could strengthen the awaking role. In our trial, strict quality control procedures have been applied to avoid bias, such as randomization and allocation concealment, assessor blinding and adequate sample size. However, methodological limitations still exist. Due to the nature of acupuncture manipulation, the therapists and the participants cannot be blinded in this study.

In this study, the acupuncture points of PC6ロGV26 and SP6 were selected based on Shixuemin's Xingnaokaiqiao theory[30]. Xingnaokaiqiao method has been applied by many clinical doctors to treat mental diseases and reported satisfactory clinical curative effects[31-32]. PC6 is an acupoint mainly used to treat mind-mental disorders and heart diseases in traditional Chinese medicine. It has been proved that PC6 acupuncture could increase connections between cerebral cortex regions[33]. GV26 also plays a positive role in recovery of neurological function after stroke. Animal research has indicated that electroacupuncture at GV26 improves neurological function in rats following cerebral ischemia/reperfusion injury, and may be associated with down-regulation of BKCa channel protein and mRNA expression[34]. fMRI studies revealed that acupuncture at SP6 could induce changes in neural activity in the sensorimotor cortical network of a sleep-deprived brain[35]. Auricular acupuncture (AA) is a safe nonpharmaceutical complementary medicine technique based on a hypothesis that the entire human body is represented on the external auricle[36]. The superiority of AA has been examined in many disorders, such as pain[37], constipation[38], insomnia[39] and congnitive impairment[40]. The auricular point "Heart" is located in the middle of the ear armor cavity and is closely related with consciousness according to the traditional Chinese theory "Heart Govern the Spirit Light". Previous studies have certified that stimulation at auricular point "Heart" and "Brainstem" could have positive effect on brain function[41-43]. It is worth mentioning that the position of the two points are partially coincident with the distribution of the auricular vagal nerve, which is considered to be a mechanism behind the analgesic effects of auricular acupuncture[44-45]. 
In conclusion, this study will provide solid evidence of the role of combined application of auricular and body acupuncture in improving the consciousness of patients after TBI.

\section{Abbreviations}

DOC: Disorder of consciousness; GCS: Glasgow Coma Scale; MMN: Mismatch negativity; TBI: Traumatic brain injury

\section{Declarations}

Trial status

This is protocol version1.0, version date is 2018.9.19. Participants are being recruited. Recruitment began on 1 June 2018. The trial is planned to be completed by 30 June 2020.

Acknowledgments

We are thankful to Prof. Nie Bin, GuangDong Second Hospital of Traditional Chinese Medicine, for scientific designment.

\section{Funding}

This report is financially supported by the National Science Foundation for Young Scientists of China, No. 81704179.

Availability of data and materials

The results of this trial will be presented through scientific reports or journals.

Authors' contributions $\rrbracket$

LT and LY: designed the study protocol and wrote the manuscript; KW and WXY: acupuncture treatment $\triangle$ WX and JY: outcome assessment; YJ: statistical analysis; QXJ: ethical approval; ZY and ZH: follow-up. All authors approve the final version of the paper. The corresponding author has the sole responsibility of submission of the manuscript for publication.

Ethics approval and consent to participate

We strictly follow the principles of the medical ethics of the Declaration of Helsinki with the approval of the Ethics and Research Committee of GuangDong Second Hospital of Traditional Chinese Medicine, China.llf there are any protocol modifications, we will report to the Ethics and Research Committee for approval. All patients will be recruited from the Department of Acupuncture and Rehabilitation, GuangDong Second Hospital of Traditional Chinese Medicine. Informed consent will be obtained from all 
study participants and their legal guardians. Participant information will be protected. All experimental data will be stored in a secure storage area with access limited to the researchers alone.

Consent for publication

Not applicable.

Competing interests

The authors declare that they have no competing interests.

\section{References}

[1] Maas, Andrew I R, Stocchetti, et al. Moderate and severe traumatic brain injury in adults[J]. Lancet Neurology, 2008, 7(8):728-741.

[2] LiY, GuJ, ZhouJ, et al. The epidemiology of traumatic brain injury in civilian inpatients of Chinese Military Hospitals, 2001-2007. Brain Inj. 2015;29:981-8.

[3] Liu J , Xue X, Wu Y, et al. Efficacy and safety of electro-acupuncture treatment in improving the consciousness of patients with traumatic brain injury: study protocol for a randomized controlled trial[J]. Trials, 2018, 19(1):296.

[4] Kinney HC, Samuels MA. Neuropathology of the persistent vegetative state. A review[J]. Journal of Neuropathology \& Experimental Neurology, 1994, 53(6):548-58.

[5] Adams JH, Jennett B, Mclellan DR, et al. The neuropathology of the vegetative state after head injury[J]. Journal of Clinical Pathology, 1999, 52(11):804-806. \

[6] Adams JH, Jennett B, Mclellan DR, et al. The neuropathology of the vegetative state after head injury[J]. Journal of Clinical Pathology, 1999, 52(11):804-806.

[7] McDonald CM, Jaffe KM, Fay GC, et al. Comparison of indices of traumatic brain injury severity as predictors of neurobehavioral outcome in children. Arch Phys Med Rehabil. 1994; 75(3):328-37.

[8] Austin CA, Slomine BS, Dematt EJ, et al. Time to follow commands remains the most useful injury severity variable for predicting WeeFIM(R) scores 1 year after paediatric TBI. Brain Inj. 2013; 27(9):105662.

[9] Pangilinan PH, Giacoletti-Argento A, Shellhaas R, et al. Neuropharmacology in pediatric brain injury: a review. PMR. 2010; 2(12):1127-40. [10] Patrick PD, Wamstad JB, Mabry JL, Smith-Janik S, Gurka MJ, Buck ML, et al. Assessing the relationship between the WNSSP and therapeutic participation in adolescents in low response states following severe traumatic brain injury. Brain Inj. 2009; 23(6):528-34. 
[11] Xu KS, Song JH, Huang TH, et al. Clinical eficacy observation of acupuncture at Suliao(GV 25)on improving regain of consciousnes from coma in severe craniocerebral injury[J]. Zhongguo Zhen Jiu, 2014, 34(6):529-533.

[12] Song $H$, Chen $X, Y u Y$, et al. Xingnao Kaiqiao acupuncture combined with Angong Niuhuang Wan for a patient under persistent vegetative state: a case report[J]. Frontiers of Medicine, 2018, 12(3):1-6.

[13] Murakami M, Fox L, Dijkers MP. Ear acupuncture for immediate pain relief-A systematic review and meta-analysis of randomized controlled trials. Pain Medicine. 2017;18(3):551-564.

[14] Yang LH, Duan PB, Du SZ, et al. Efficacy of auriculotherapy for constipation in adults: A systematic review and meta-analysis of randomized controlled trials. The Journal of Alternative and Complementary Medicine. 2014;20(8):590-605.

[15] Baker TE, Chang G. The use of auricular acupuncture in opioid use disorder: A systematic literature review. American Journal on Addictions. 2016;25(8):592-602.

[16] Garner BK, Hopkinson SG, Ketz AK, et al. Auricular Acupuncture for Chronic Pain and Insomnia: A Randomized Clinical Trial[J]. Acupuncture in Medicine, 2018, 30(5):262-272.

[17] Kwon CY, Lee B, Suh HW, et al. Efficacy and Safety of Auricular Acupuncture for Cognitive Impairment and Dementia: A Systematic Review[J]. 2018.

[18] Schulz KF, Altman DG, Moher D. CONSORT 2010 statement: updated guidelines for reporting parallel group randomized trials. BMC Med. 2010;8:18.

[19] James KE, Bloch DA, Lee KK, et al. An index for assessing blindness in a multi-centre clinical trial: disulfiram for alcohol cessation-a VA cooperative study. Stat Med. 1996;15:1421-34.

[20] Auricular acupoint Diagnosis and Treatment Professional Committee of China Acupuncture Society. National standard of the People's Republic of China "Auricular Acupoint name and Positioning" (GB/T13734-2008) [S]. Beijing: China Standards Press, 2008.

[21] Lim S. WHO Standard Acupuncture Point Locations[J]. Evidence-based Complementary and Alternative Medicine: eCAM, 2010, 7(2):167-168.

[22] Watzlawick R, Howells D W, Schwab J M. Neuroprotection After Traumatic Brain Injury[J]. Jama Neurology, 2015, 73(2):149-150.

[23] Jenkins P O, Mehta M A, Sharp D J. Catecholamines and cognition after traumatic brain injury[J]. Brain, 2016, 139(9):2345-2371.

[24] Taylor CA, Bell JM, Breiding MJ, et al. Traumatic Brain Injury-Related Emergency Department Visits, Hospitalizations, and Deaths - United States, 2007 and 2013.[J]. Morbidity \& Mortality Weekly Report 
Surveillance Summaries, 2017, 66(9):1.

[25] Joseph B, Haider A, Rhee P. Traumatic brain injury advancements.[J]. Current Opinion in Critical Care, 2015, 21(6):506.

[26] Majdan M, Plancikova D, Brazinova A, et al. Epidemiology of traumatic brain injuries in Europe: a cross-sectional analysis.[J]. Lancet Public Health, 2016, 1(2):e76.

[27] Cheng P, Yin P, Ning P, et al. Trends in traumatic brain injury mortality in China, 2006-2013: A population-based longitudinal study[J]. Plos Medicine, 2017, 14(7):e1002332.

[28] Matsumotomiyazaki J, Asano Y, Yonezawa S, et al. Acupuncture Increases the Excitability of the Cortico-Spinal System in Patients with Chronic Disorders of Consciousness Following Traumatic Brain Injury[J]. Journal of Alternative \& Complementary Medicine, 2016, 22(11) :887-894.

[29] Matsumoto-Miyazaki J, Asano Y, Ikegame Y, et al. Acupuncture Reduces Excitability of Spinal Motor Neurons in Patients with Spastic Muscle Overactivity and Chronic Disorder of Consciousness Following Traumatic Brain Injury[J]. J Altern Complement Med, 2016, 22(11):895-902.

[30] Xingnaokaiqiao Method. In: Shi XM. Chief Editor. Shi Xuemin Practical acupuncture. 1st ed. Beijing: Traditional Chinese Medicine Press; 2009. p. 264-76.

[31] Yang ZX, Xie JH, Liu DD, et al. Xingnao Kaiqiao needling method for acute ischemic stroke: a metaanalysis of safety and efficacy[J]. Neural Regeneration Research, 2017, 12(8):1308-1314.

[32] Song $\mathrm{H}$, Chen $\mathrm{X}, \mathrm{Yu} \mathrm{Y}$, et al. Xingnao Kaiqiao acupuncture combined with Angong Niuhuang Wan for a patient under persistent vegetative state: a case report[J]. Frontiers of medicine, 2018 Jun;12(3):334339.

[33] Yu H, Xu G, Guo L, et al. Magnetic stimulation at Neiguan(PC6) acupoint increases connections between cerebral cortex regions[J]. Neural Regeneration Research, 2016, 11(7):1141-1146.

[34] Wang Y, Shen Y, Lin HP, et al. Large-conductance Ca (2+)-activated K + channel involvement in suppression of cerebral ischemia/reperfusion injury after electroacupuncture at Shuigou(GV26) acupoint in rats[J]. Neural Regeneration Research, 2016, 11(6):957-962.

[35] Gao L, Zhang M, Gong H, et al. (2014). Differential activation patterns of FMRI in sleep-deprived brain: restoring effects of acupuncture. Evid Based Complement. Alternat Med. 2014:465760.

[36] Usichenko T, Anders E. Auricular acupuncture. In: Filshie White, Cummings, editors. Medical acupuncture: a western scientific approach. second ed. Elsevier; 2016.

[37] Murakami M, Fox L, Dijkers MP. Ear Acupuncture for Immediate Pain Relief-A Systematic Review and Meta-Analysis of Randomized Controlled Trials[J]. Pain Medicine, 2016, 18(3):215. 
[38] Yang LH, Duan PB, Duan SZ, et al. Efficacy of auriculotherapy for constipation in adults: a systematic review and meta-analysis of randomized controlled trials[J]. Journal of Alternative \& Complementary Medicine, 2015, 58(2):30.

[39] Lan Y, Wu X, Tan H J, et al. Auricular acupuncture with seed or pellet attachments for primary insomnia: a systematic review and meta-analysis.[J]. Bmc Complementary \& Alternative Medicine, 2015, 15(1):1-14.

[40] Chan-Young K, Boram L, Hyo-Weon S, et al. Efficacy and Safety of Auricular Acupuncture for Cognitive Impairment and Dementia: A Systematic Review[J]. Evidence-Based Complementary and Alternative Medicine, 2018, 2018:1-15.

[41] Romoli M, Allais G, Airola G, et al. Ear acupuncture and fMRl: a pilot study for assessing the specificity of auricular points[J]. Neurological Sciences, 2014, 35(1 Supplement):189-193.

[42] Zhao L, Zhang L, Bao H, et al. Correlation between positive reaction point of auricular points and stroke.[J]. Zhongguo Zhen Jiu, 2015, 35(6):609-612.

[43] Shan QH, Han YD, Yin JH, et al. Effect of electroacupuncture at auricular acupoint heart area for cardiac and cerebrovascular function [J]. Zhongguo Zhen Jiu, 1996(9):9-11.

[44] Usichenko T, Hacker H, Lotze M. Transcutaneous auricular vagal nerve stimulation (taVNS) might be a mechanism behind the analgesic effects of auricular acupuncture.[J]. Brain Stimulation, 2017, 10(6):1042.

[45] Peng L, Mu K, Liu A, et al. Transauricular vagus nerve stimulation at auricular acupoints Kindey (C010), Yidan (CO11), Liver (CO12) and Shenmen (TF4) can induce auditory and limbic cortices activation measured by fMRI.[J]. Hear Res, 2017, 359.

\section{Tables}

Table1 Description of the applied acupuncture points

\begin{tabular}{ll}
\hline Acupoints & Descriptions \\
\hline GV26: Shuigou & at the junction of the upper and middle third of the philtrum \\
$\begin{array}{l}\text { PC6: Neiguan } \\
\text { longus and m. flexor radialis } \square\end{array}$ \\
$\begin{array}{l}\text { SP6:Sanyinjiao } \\
\begin{array}{l}\text { Auricular acupoint } \\
\text { "Heart" }\end{array}\end{array}$ \\
$\begin{array}{l}\text { Auricular acupoint } \\
\text { "Brainstem" }\end{array}$
\end{tabular}




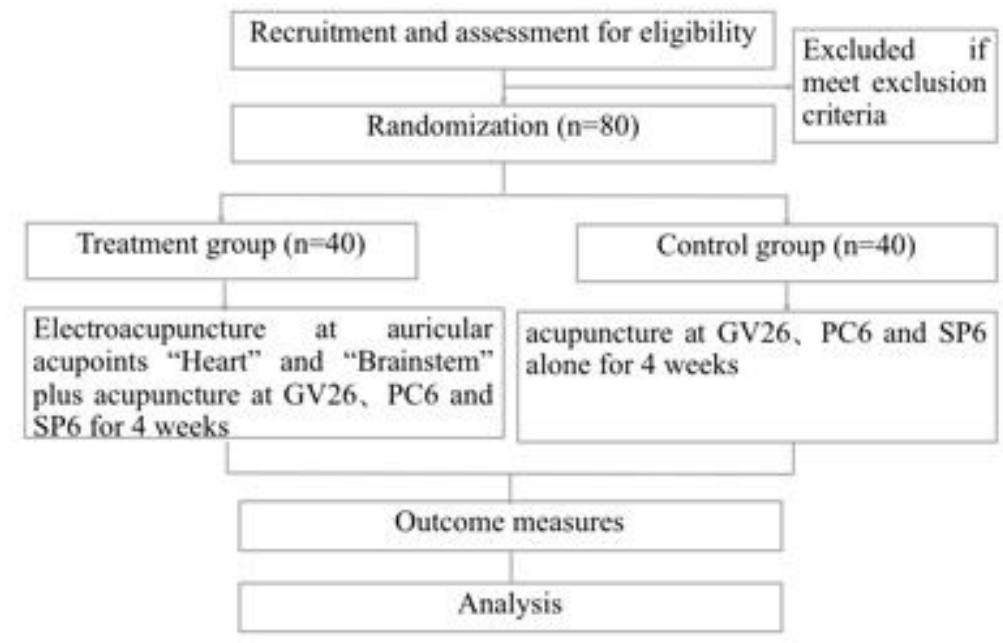

\section{Figure 1}

Flow chart of the study procedure.

\begin{tabular}{|c|c|c|c|c|c|c|c|c|c|}
\hline & & \multicolumn{8}{|c|}{ Study period } \\
\hline & \multirow{3}{*}{$\begin{array}{l}\text { Week } \\
\text { Visit }\end{array}$} & \multirow{2}{*}{$\begin{array}{c}\text { Enrolment } \\
-t 1\end{array}$} & \multirow{2}{*}{$\begin{array}{l}\text { Allocation } \\
\text { to }\end{array}$} & \multicolumn{5}{|c|}{ Postallocation } & \multirow{3}{*}{$\begin{array}{c}\text { Close-out } \\
12 \\
21\end{array}$} \\
\hline \multirow{2}{*}{$\begin{array}{l}\text { Time } \\
\text { point* }\end{array}$} & & & & \multirow{2}{*}{$\frac{1}{1-5}$} & \multirow{2}{*}{$\frac{2}{6-10}$} & \multirow{2}{*}{$\frac{3}{11-15}$} & \multicolumn{2}{|c|}{4} & \\
\hline & & $-t 1$ & to & & & & $16-19$ & 20 & \\
\hline \multirow{4}{*}{\multicolumn{2}{|c|}{$\begin{array}{l}\text { ENROLMENT; } \\
\text { Eligibility screen } \\
\text { Informed consent } \\
\text { Allocation }\end{array}$}} & & & & & & & & \\
\hline & & $x$ & & & & & & & \\
\hline & & $x$ & & & & & & & \\
\hline & & & $x$ & & & & & & \\
\hline \multirow{2}{*}{\multicolumn{10}{|c|}{$\begin{array}{l}\text { Interventions: } \\
\text { Treatment group }\end{array}$}} \\
\hline & & & & & & & & $\rightarrow$ & \\
\hline \multicolumn{10}{|c|}{ Control group } \\
\hline \multicolumn{10}{|c|}{ Assessment: } \\
\hline GCS & & $x$ & $x$ & & & & & $\rightarrow$ & $x$ \\
\hline $\mathrm{MMN}$ & & & $\times$ & & & & & $x$ & \\
\hline $\mathrm{ADL}$ & & & $\times$ & & & & & $x$ & $\times$ \\
\hline FuglM & & & $x$ & & & & & $x$ & $x$ \\
\hline Safety & & & & & & & & $\rightarrow$ & \\
\hline
\end{tabular}

\section{Figure 2}

Schedule of enrollment, interventions, and assessments.

\section{Supplementary Files}

This is a list of supplementary files associated with this preprint. Click to download.

- supplement1.doc 